\title{
Evolutionary Algorithms Applied to Lens Design: Case Study and Analysis
}

\author{
Simon Thibault ${ }^{\mathrm{a}}$, Christian Gagné ${ }^{\mathrm{b}}$, Julie Beaulieu ${ }^{\mathrm{b}}$, Marc Parizeau $^{\mathrm{b}}$ \\ ${ }^{a}$ ImmerVision, 2020, University, Montreal, Quebec, H3A 2A5, Canada \\ ${ }^{\mathrm{b}}$ Laboratoire de Vision et Systèmes Numériques (LVSN), Département de Génie Électrique et \\ de Génie Informatique, U. Laval, Québec (Québec), G1K 7P4, Canada
}

\begin{abstract}
Lens system design makes extensive use of optimization techniques to improve the performance of an optical system. We know that designing a lens system is a complex task currently done by experienced optical designers, using specialized optical design software tools. In order to contribute to this particular field, this paper presents a comparison between lens design done by optical designers and evolutionary algorithms lens based design. Evolutionary algorithms consist in population-based global search methods inspired by natural evolution. They are recognized to be particularly efficient for complex non-linear optimization problems. Given the non-linear nature of lens design as an optimization process, evolutionary algorithms are good candidates for automating this task. The evolutionary algorithms were applied to the monochromatic quartet that was presented to expert participants at the International Lens Design Conference in 1990 (a friendly competition). Comparative results demonstrate that the evolutionary approach is able to find solutions slightly better than those presented at the competition. Then a real-life imaging problem is tackled. Results show that an evolutionary algorithm is again able to discover lens systems comparable to design done after a reasonable effort by experts. This paper presents an analysis of this approach for automatic lens design from a real-life optical design point of view.
\end{abstract}

Keywords: Optimization, evolutionary algorithms, lens design.

\section{INTRODUCTION}

Usually, modern lens system design is conducted using specialized CAD tools that help designers to visualize the lens system, to evaluate its quality based on precise criteria, and to locally optimize the system variables. The design space of optical systems is typically a complicated multidimensional parameter space. By constructing a merit function (MF) that expresses the departure of individual configurations from ideal required performance, it is possible for the optical designer to determine all of the possible configurations that will yield the best solution to the design problem. Using a human based design procedure, an optical designer chooses an initial configuration and with the help of an automatic optimization algorithm modifies the configuration, moving it around in the design space to find a solution that gives the best, or, at least, acceptable performance. Even the best optimization routines cannot, in general, find the global optimum, the local minimum of the MF can be a very good solution based on the optical performances but also in terms of cost and manufacturing.

Most of the popular optimization routines like Damped Least Squares (DLS) are based on a gradient descent principle that only accepts steps that decrease the merit function. Thus the designer's solution often gets trapped in a local minimum ${ }^{1}$. This fact has motivated the development of global optimization (GO) tools. Today, computing power is available and more and more optical designers will use GO algorithms for maximizing the performance of their design. Several techniques are available. Many inexperienced optical designers will use a global search algorithm starting from a very rough initial design. Very rough means that the design has the correct number of surfaces, a defined stop position, initial glass selection, field of view, and wavelength. Sometimes, the very rough initial configuration can be parallel plates of glass with a curvature solved on the last surface to control the focal length. By defining a MF as well as many variables, the global search algorithms implemented in commercial $\mathrm{CAD}$ tools will find promising design forms but not necessarily the best possible solution. This latter job is left to the optical designer or to a separate algorithm. Generally, the global search will generate many solutions. Because the lowest merit function is not always the best (cost and fabrication issues), the designer will want to investigate one or two promising optical systems. By using those systems as a starting point, the optical designer will refine 
them by making adjustments and optimizations. This can be done by hand or by other algorithms like Hammer Optimization $^{2}$. Finally, the best optical design is the one selected by the optical designer at the end. The choice will be motivated by a lot of factors such as potential cost, manufacturing, assembly, procurement and personal feeling. The art of optical design with computer assistance is to be able to determine a good starting point and supervise the optimisation process. An experienced optical designer knows when a program must be coaxed into a more fruitful direction.

\section{LENS SYSTEM DESIGN WITH EVOLUTIONARY ALGORITHMS}

The typical search space of optical system design is a complicated multidimensional space comprising several peaks, non-linearity and strong correlation between parameters ${ }^{3}$. We know that a local search explores only the immediate neighborhood of the initial solution, making the result very dependent on the starting point solution. Since the end of the 1980's, several applications of global search methods have been made in optical design. More popular optical software now incorporates many simulated annealing or specialized algorithms to allow exploration beyond optima ${ }^{4-6}$.

Evolutionary Algorithms (EA) are black-box population-based optimization algorithms inspired by natural evolution. They consist in evolving a population of solutions to a problem represented by an appropriate data structure (i.e. bit strings, real-valued vectors or computer programs) on which natural selection biased toward the "fittest" solutions and genetic operations such as crossover and mutation are applied. EA are now recognized as global optimization algorithms capable of dealing well with the exploration of hard non-linear, multi-modal functions and/or multi-objective optimization. On the other hand, their principal disadvantages are: 1) they cannot provide convergence guarantees, given their stochastic nature, and 2) the high computational cost required to evaluate the quality of millions, if not billions, of solutions required for the adequate optimization of hard problems.

The idea of using EA as a global optimization method for lens system design has been investigated by several researchers ${ }^{7-8}$. Our recent work $^{8}$ has shown that evolutionary computations can be human competitive for real world lens design. The algorithms used were able to repeatedly generate excellent solutions to two different problems tackled with specific constraints and optimization criteria, including some difficult mechanical restrictions. In some cases, results have even surpassed those obtained by human experts, at least for the used criteria. In the following, we analyze these results further from a real-life optical design point of view.

\section{CASE STUDY}

\subsection{Monochromatic Quartet}

Every four years, optical designers around the world can meet at an international optical or lens design conference. A lens design problem is always proposed in advance of the meeting so that attendees can work on this problem. Attendee solutions can be discussed and covered during the meeting and in a paper. Because the problem is always original and challenging, a large number of participants produce designs which can later serve as benchmark baselines for evaluating the performance of optimization processes.

The Monochromatic Quartet was defined for the 1990 International Lens Design Conference (ILDC now IODC) ${ }^{9}$. The statement of the problem is as follows: 
Désign a 4-element, $f / 3,100 \mathrm{~mm}$ effective focal length lens of BK7 glass, illuminated by helium $d$ wavelength (i.e., $n=1.51680$ ). The object is at infinity, the object field covers $30^{\circ}$ full field $\left(15^{\circ}\right.$ semi-field angle) and the image field is flat.

Constraints on the construction included: only spherical surfaces, no aspherics, GRIN elements, Fresnel lenses, binary elements, holographic optical elements, etc. The minimum glass thickness is $2 \mathrm{~mm}$, but there was no upper limit on the size of the lens. The distortion must be less than $1 \%$ and there was to be no vignetting. The last was intended to assure that vignetting could not be used to improve the edge performance on the lens. No requirement was put on the location of the stop of the system. equally.

The merit function consisted of the average of the RMS blur spot for three fields: on-axis, $10.5^{\circ}$, and $15^{\circ}$, weighted

Figure 1 shows two of the best lenses achieved by 1990 conference participants. We will compare these two lenses with two lenses found by EA. Figure 2 presents the two lenses found using two different types of EA. Figure 2A shows a lens found by using a non-isotropic Self-Adaptive Evolution Strategy (SA-ES) ${ }^{10}$, and Figure 2B shows a lens found by a Covariance Matrix Adaptation Evolution Strategy (CMA-ES) ${ }^{11}$.

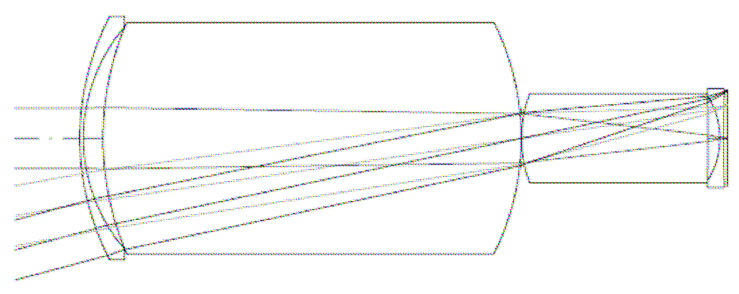

\begin{tabular}{|c|c|c|c|}
\hline $\begin{array}{c}\text { Radius } \\
(\mathrm{mm})\end{array}$ & $\begin{array}{c}\text { Thickness } \\
(\mathrm{mm})\end{array}$ & $\begin{array}{c}\text { Semi- } \\
\text { Aperture }\end{array}$ & Glass \\
\hline 0.0 & $\infty$ & - & AIR \\
\hline 145.6875 & 2.0 & 64.9691 & BK7 \\
\hline 94.7116 & 10.8039 & 61.6892 & AIR \\
\hline 162.7274 & 231.4971 & 61.6778 & BK7 \\
\hline-143.6539 & 0.0 & 15.3583 & AIR \\
\hline 0.0 & 0.1 & 13.5674 & AIR \\
\hline 68.4004 & 103.2294 & 14.3693 & BK7 \\
\hline-1480.2792 & 6.7215 & 23.2095 & AIR \\
\hline-43.0337 & 2.0 & 23.2221 & BK7 \\
\hline 858.3420 & 2.0046 & 25.8911 & AIR \\
\hline 0.0 & - & 26.7948 & - \\
\hline
\end{tabular}

(Bold surface is the aperture stop.)

a)

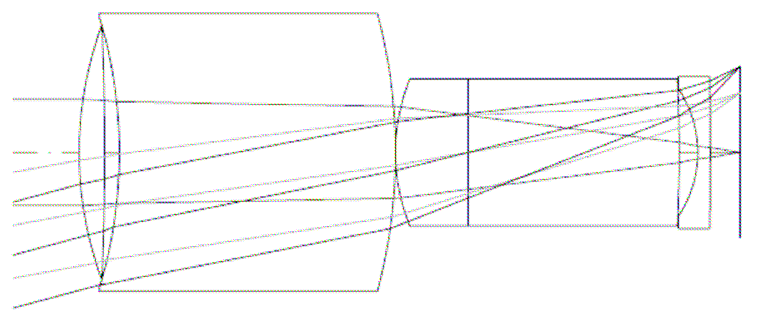

\begin{tabular}{|c|c|c|c|}
\hline $\begin{array}{c}\text { Radius } \\
(\mathrm{mm})\end{array}$ & $\begin{array}{c}\text { Thickness } \\
(\mathrm{mm})\end{array}$ & $\begin{array}{c}\text { Semi- } \\
\text { Aperture }\end{array}$ & Glass \\
\hline \hline 0.0 & $\infty$ & - & AIR \\
\hline 116.753325 & 8.0 & 41.3577 & BK7 \\
\hline-896.302517 & 4.6 & 41.5202 & AIR \\
\hline-148.252719 & 86.666308 & 41.7531 & BK7 \\
\hline-170.784756 & 0.0 & 23.9590 & AIR \\
\hline 61.852545 & 22.856198 & 21.7151 & BK7 \\
\hline 0.0 & 66.187961 & 11.5910 & BK7 \\
\hline-406.404293 & 5.8 & 19.5193 & AIR \\
\hline-36.156468 & 3.965152 & 19.5915 & BK7 \\
\hline-1470.14649 & 0.0 & 22.4919 & AIR \\
\hline 0.0 & - & 26.7948 & - \\
\hline
\end{tabular}

(Bold surface is the aperture stop.)

b)

Figure 1: Best of two design classes presented by human experts for the monochromatic quartet problem: a) solution 14; and b) solution 7 .

The four lenses have been analyzed using Zemax-EE software (Nov. 2004 version) ${ }^{12}$. The objective of the analysis was not to compare the image quality (spot size), which has been discussed in related publications, but to focus on some other aspects of real-life lens design. We have looked through to Seidel coefficients, Optical Path Difference (OPD), the spot size out of the field (middle points) and tolerances. 
Table 1 shows the Seidel coefficients for the design presented in Figures 1 and 2. The Seidel coefficients are the sum for the entire system. The coefficients listed are for spherical aberration (SPHA), coma (COMA), astigmatism (ASTI), field curvature (FCUR) and distortion (DIST).

Table 1: Seidel sum for various lenses. (in $\mathrm{mm}$ )

\begin{tabular}{|c|c|c|c|c|c|}
\hline Lens id & Spherical aberration & Coma & Astigmatism & Field curvature & Distortion \\
\hline CMA-ES & 0.00375 & -0.00208 & 0.00149 & 0.00796 & 0.05253 \\
\hline SA-ES & 0.00319 & -0.00167 & -0.00430 & 0.00810 & 0.02953 \\
\hline Solution 7 & 0.00626 & -0.00236 & 0.00597 & 0.00300 & -0.09902 \\
\hline Solution 14 & 0.00555 & -0.00132 & 0.00288 & 0.00207 & 0.01043 \\
\hline
\end{tabular}

As we can see, the two lenses found by EA present a lower spherical aberration but the field curvature is also significantly larger than lenses found by the human experts. In a sense all those lenses are good based on the lens design criteria. The OPD shape and value show that the EA lenses are not well corrected for marginal rays (rays closer to the edge of the pupil) but very well corrected for central rays (ray closer to the chef ray). Figure 3 gives the RMS spot size for the entire FOV, not only at three specific values. It shows clearly that the human design presents a more uniform value over the whole field.

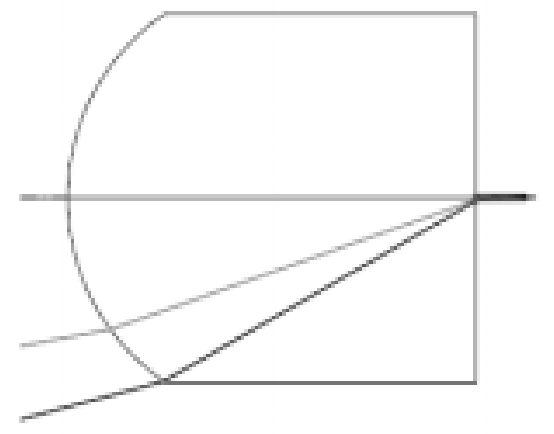

\begin{tabular}{|c|c|c|c|}
\hline $\begin{array}{c}\text { Findias } \\
\text { (mmi) }\end{array}$ & $\begin{array}{l}\text { Thickness } \\
\text { (man) }\end{array}$ & $\begin{array}{l}\text { Secti- } \\
\text { Apcorture }\end{array}$ & CInos \\
\hline 0.0 & $\infty$ & $=$ & AIR \\
\hline 11141.72268 & 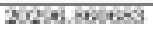 & श15.5313 & BEF \\
\hline $184.3 \mathrm{k} 50$ & 110.755600 & 153.1524 & AIIR \\
\hline-487.56500 & 2438206056 & 151.970 & BET \\
\hline 0.0 & 5.W6Eस & 49.4441 & BKF \\
\hline-206.80164 & 8.374215 & 49.4441 & AIIR \\
\hline 224.63126 & A.ASITVO & 4H.A17\% & BKT \\
\hline Isisctevels & Sachos & AFA147 & ATR \\
\hline$-288.6 \mathrm{ETH}$ & 20004056 & 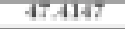 & BKT \\
\hline-2358900 & มा117า & 45.ses5 & ATा \\
\hline $0 \%$ & $=$ & 26.7056 & $=$ \\
\hline
\end{tabular}

a)

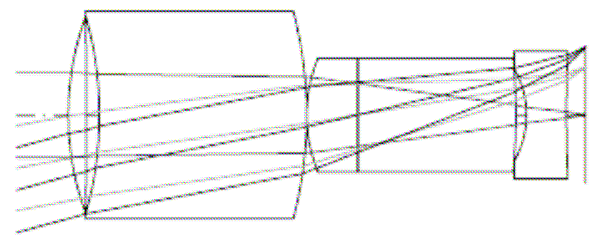

\begin{tabular}{|c|c|c|c|}
\hline $\begin{array}{c}\text { Radius } \\
\text { (mm) }\end{array}$ & $\begin{array}{c}\text { Thickness } \\
\text { (mm) }\end{array}$ & $\begin{array}{c}\text { Semi- } \\
\text { A perture }\end{array}$ & Glass \\
\hline 0.0 & 0 & & AIR \\
\hline 112.73243 & 7.052794 & 38.8061 & BK7 \\
\hline-4616.30240 & 5.132246 & 38.8061 & AIR \\
\hline-144.83956 & 81.537700 & 38.8061 & BK7 \\
\hline-160.81318 & -0.000000 & 23.0086 & AIR \\
\hline 57.83538 & 20.009066 & 20.8839 & BK7 \\
\hline 0.0 & 62.313838 & 12.2913 & BK7 \\
\hline-216.40247 & 4.115601 & 18.3684 & AIR \\
\hline-36.90077 & 15.687862 & 18.3684 & BK7 \\
\hline 492.37558 & 7.557047 & 23.6293 & AIR \\
\hline 0.0 & - & 26.7949 & - \\
\hline \multicolumn{4}{|c|}{ Bold surface is the aperture stop.) } \\
\hline
\end{tabular}

b)

Figure 2: Best lens system found with SA-ES (a) and CMA-ES (b) for the monochromatic quartet problem. 


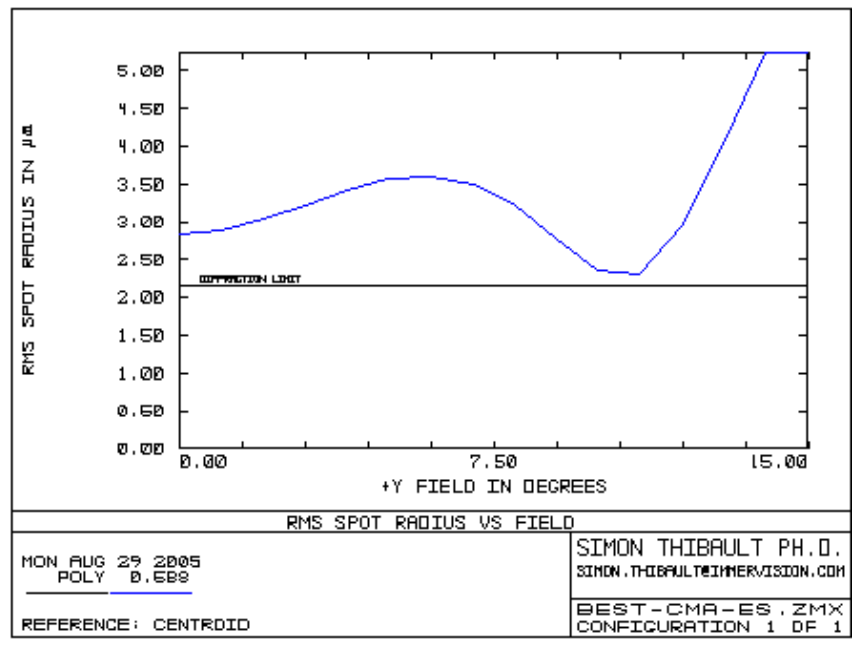

a) CMA-ES

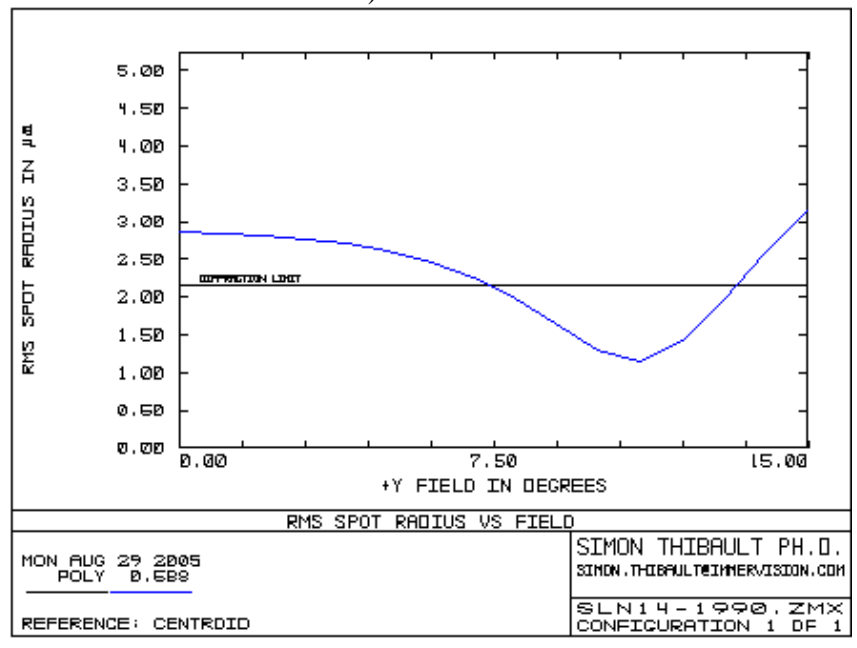

b) Solution 14

Figure 3: RMS spot size vs field of view (The horizontal lines represent the diffraction limit).

\subsection{Imaging Lens}

In order to complete the analysis done in the last section, a new lens problem was studied by both EA and human experts. This real-world application can be more instructive on the difference between human and purely EA optimization. Table 2 gives the lens specifications. This lens is much more complex because it includes many physical constraints which can be difficult to respect. 
Table 2: Imaging Lens Specifications

First order requirements

\begin{tabular}{|c|c|}
\hline \multirow{2}{*}{\multicolumn{2}{|c|}{ Obiect distance }} \\
\hline Object distance & \\
\hline F/number or NA & 4 \\
\hline Full field of View (FOV) & $80 \mathrm{~mm}$ total \\
\hline Focal length & $25 \mathrm{~mm}(\mathrm{TBD})$ \\
\hline Detector type & CCD \\
\hline Image format & 15 um X 15um, $15 \mathrm{~mm}$ \\
\hline Magnification & -0.36 \\
\hline Vignetting & $\mathrm{NO}$ \\
\hline Central wavelength & $890 \mathrm{~nm}$ \\
\hline Spectral Weight & $890 \mathrm{~nm}-1.0,865 \mathrm{~nm}-0.5,915 \mathrm{~nm}-0.5$ \\
\hline
\end{tabular}

Image quality requirements

\begin{tabular}{|l|l|}
\hline Encircled Energy & $>75 \%$ in a circle of 15 um in diameter \\
\hline
\end{tabular}

Mechanical \& Packaging requirements

\begin{tabular}{|l|l|}
\hline Length & Total track less than $44 \mathrm{~mm}$ (lens up to detector) \\
\hline Back focus distance & $>15 \mathrm{~mm}$ \\
\hline Object to image distance & $120 \mathrm{~mm}+/-10 \mathrm{~mm}$ \\
\hline Element size & $<30 \mathrm{~mm}$ in diameter \\
\hline Number of element & $<5$ \\
\hline
\end{tabular}

\subsubsection{Human Based Solution}

The lens arrangement found by the optical designer using standard optimization tools and experience was performed within the budget limits defined by the client (about 40 hours). The design is composed of five lenses within an arrangement,,,,-+-++ using SF14, LaK16A, F1, Lak23 and BASF12 glasses (Flint, Crown, flint, crown and flint) as shown in Figure 4. The ray bending is quite smooth and the lens shape is acceptable from a manufacturing point of view. No doubt that with more resources, the expert could have produced a better system.

The results mainly show that the lens is just at the limit of the specifications. The safety margin is very limited. This is the main characteristic of an optical design done by professional designers, due to limited time and money, the lens specification is just respected with a small but sufficient safety margin.

Table 3: Optical Prescription for the Human Based Solution 


\begin{tabular}{|c|c|c|c|}
\hline $\begin{array}{l}\text { Radius } \\
\text { (mm) }\end{array}$ & $\begin{array}{c}\text { Thickneses } \\
\text { (mm) }\end{array}$ & $\begin{array}{c}\text { Semi- } \\
\text { Aperture }\end{array}$ & $\begin{array}{c}\text { Glass } \\
\text { (index : v- H) }\end{array}$ \\
\hline 0.0 & 76.13582 & - & AIR \\
\hline-1259783 & 3.453205 & 4.208 & $1.762: 26.5$ \\
\hline-16.10987 & 0.249998 & 3.922 & AIR \\
\hline 18.36991 & 2.0 & 3.541 & $1.734: 51.8$ \\
\hline-32.45007 & $0.0082 \times 3$ & 3.110 & AIR \\
\hline 0.0 & $3.805-474$ & 2.497 & AIH \\
\hline-9.53878 & 2.0 & 3.523 & $1.6226: 35.7$ \\
\hline 32.39125 & 0.841538 & 4.545 & AIR \\
\hline-17.88445 & 1.2009995 & 4.503 & $1.660: 57.4$ \\
\hline-9.493337 & 025 & 5.108 & AIR \\
\hline 584.59130 & 2.046865 & 5.984 & $1.670: 39.2$ \\
\hline-26.44450 & 26.74464 & 6.327 & AIR \\
\hline 0.0 & - & 14.4 & - \\
\hline
\end{tabular}

(Bold surface is the aperture stop.)

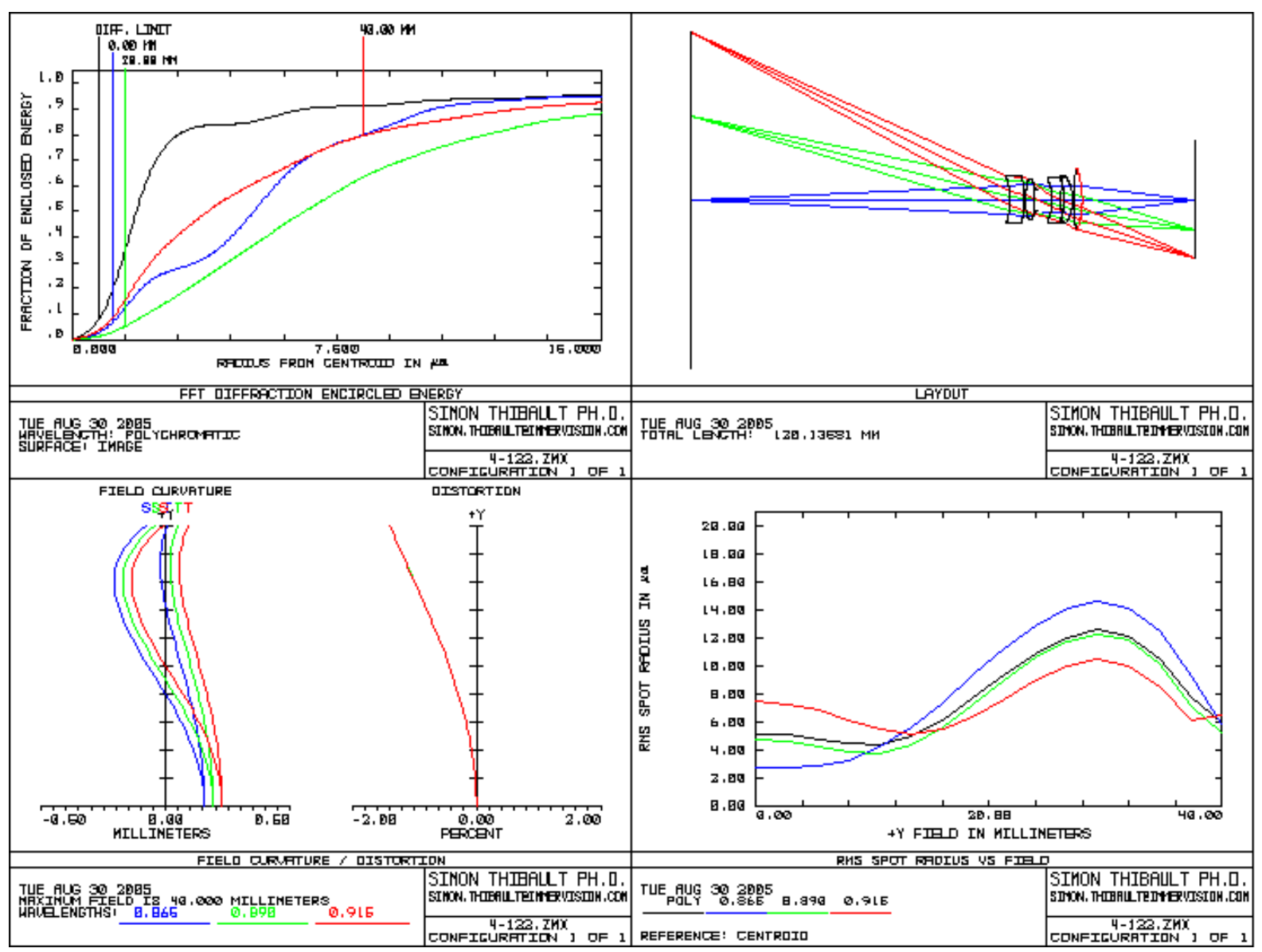

Figure 4: The solution found by human experts that respected the specifications after conducting a best effort search.

\subsubsection{EA results}

Lenses have been found by SA-ES and CMA-ES algorithms. The SA-ES solution is shown in Figure 5. The lens is composed of five lenses organized in an arrangement,,,,+--+- using flint, crown, flint, flint and flint glasses. The form of the lenses is not very uniform, the second lens is a concentric lens, and the last two are quite thick. The 
ray bending is not as smooth as the lens found by the human expert, particularly for the last lens. We also notice with the ray tracing that the pupil aberration is large (pupil marginal ray position).

Nevertheless, the performance of this design is very good on all aspects of the problem specifications. The image quality is also very good and pretty uniform along the field of view.

Table 4: Optical Prescription for the EA Solution

\begin{tabular}{|c|c|c|c|}
\hline $\begin{array}{c}\text { Radius } \\
(\mathrm{mm})\end{array}$ & $\begin{array}{c}\text { Thickness } \\
(\mathrm{mm})\end{array}$ & $\begin{array}{c}\text { Semi- } \\
\text { Aperture }\end{array}$ & $\begin{array}{c}\text { Glass } \\
\text { (index : v- } 4 \text { ) }\end{array}$ \\
\hline 0.0 & 75.0 & AIR \\
\hline 12.98357 & 2.861395 & 9.3063 & $1.744: 44.7$ \\
\hline 32.13223 & 2.690679 & 9.1445 & AIR \\
\hline 5.62296 & 0.524639 & 4.9604 & $1.755: 27.6$ \\
\hline 5.00620 & 4.615829 & 4.509 & AIR \\
\hline-19.54992 & 2.049896 & 2.589 & $1.755: 27.6$ \\
\hline-28.86507 & 3.646095 & 3.4002 & AIR \\
\hline 21.42485 & 12.345714 & 7.2616 & $1.744: 44.7$ \\
\hline-26.02713 & 8.684502 & 8.7982 & AIR \\
\hline-11.27961 & 6.481251 & 8.919 & $1.755: 27.6$ \\
\hline 3078.62227 & 0.100000 & 14.1306 & AIR \\
\hline 0.0 & - & 14.4 & - \\
\hline
\end{tabular}

(Bold surface is the aperture stop.) 


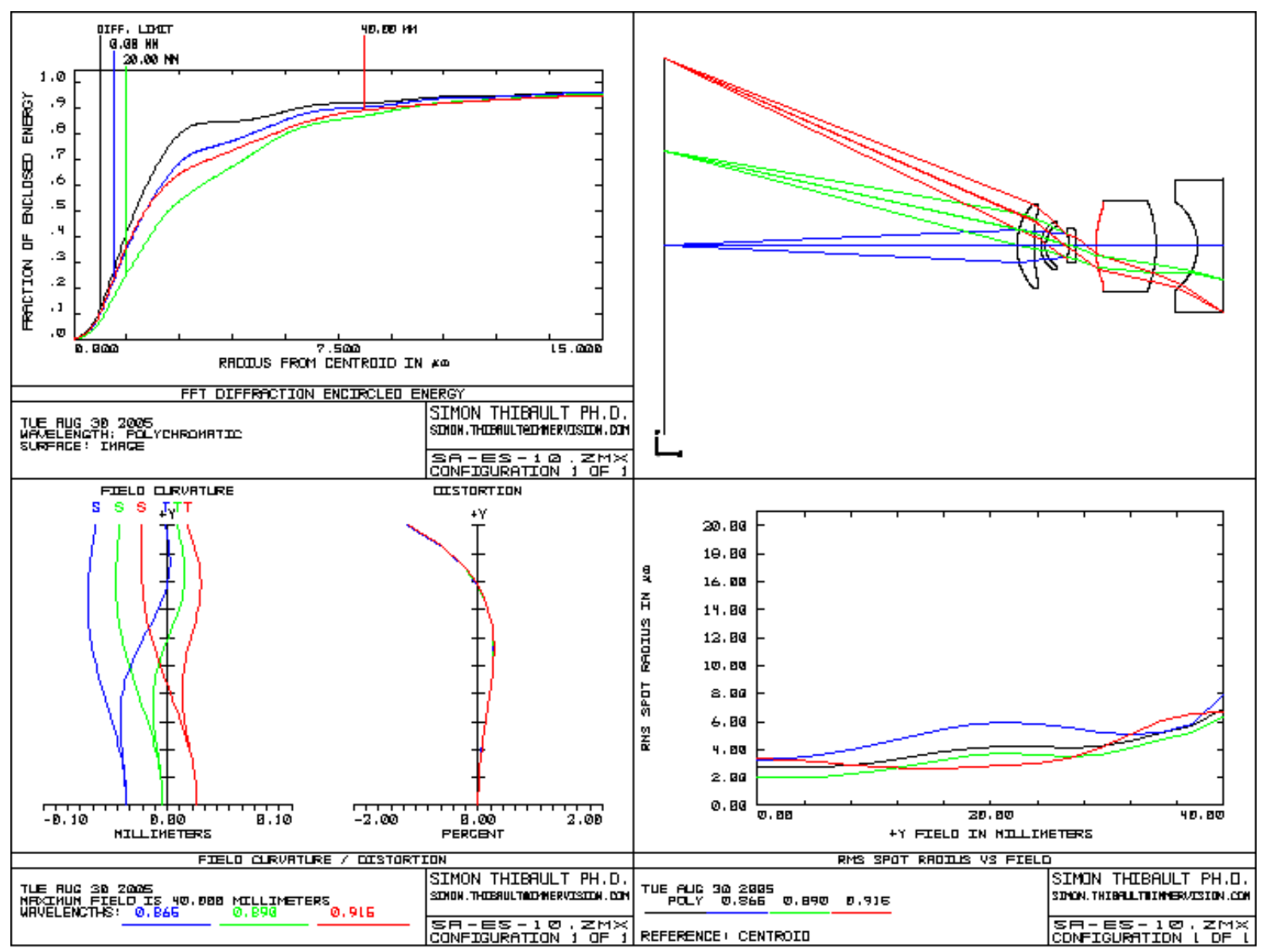

Figure 5: Best lens system found with non-isotropic SA-ES for imaging lens system problem.

\subsubsection{Tolerancing}

One of the most important criteria for a real-world design, however, is the manufacturing and the assembly of the lenses. Tolerancing a lens consists of these steps (see Zemax Manual for details ${ }^{12}$ ):

1) Define an appropriate set of tolerances for the lens. Table 5 gives the set of tolerances.

Table 5 : Optics manufacturing tolerances

\begin{tabular}{|l|c|}
\hline Attribute & Quality \\
\hline Glass quality (nd)/Vnumber & $+/-0.001 / 1.0$ \\
\hline Center Thickness (mm) & $+/-0.100$ \\
\hline Power-Irregularity (fringe) & $4-1$ \\
\hline Wedge Lens (ETD, mm) & $+/-0.050$ \\
\hline Lens Decenter (mm) & $+/-0.1$ \\
\hline Air Thickness (mm) & $+/-0.1$ \\
\hline
\end{tabular}

2) Back focal distance is used as compensators with a $5 \mathrm{~mm}$ range. 
3) Select an appropriate criterion, the RMS spot radius is used.

4) Sensitivity mode is used.

5) Worst offenders are recorded.

6) Monte Carlo (MC) Analysis: The sensitivity analysis considers the effects on system performance for each tolerance individually. The aggregate performance is estimated by a root-sum-square calculation. As an alternative way of estimating aggregate effects of all tolerances, a Monte Carlo simulation was performed. This simulation generates a series of random lenses, which meets the specified tolerances, then evaluates the criteria. No approximation is made other than the range and magnitude of defects considered. By considering all applicable tolerances simultaneously and exactly, a highly accurate simulation of the expected performance is possible. The Monte Carlo simulation generates 20 designs, using normal statistic distribution for each tolerance attribute.

The results are as follows:

\section{Human based design:}

The worst offender is the decenter of the third lens (just after the stop) and it produces a change in the RMS spot size of $109 \%$. The statistical analysis (20 trials) shows that the best system raises the spot by $158 \%$, the worst by $500 \%$, with an average increase of $300 \%$. The RMS spot size after manufacturing will be less than 32 um with a probability of $90 \%$, less than 25 um with $50 \%$, and $10 \%$ of the lens will have a RMS spot size less than 13 um.

\section{EA lens:}

The worst offender is the thickness of the second lens (concentric lens) and it produces a change in the RMS spot size of $1500 \%$. The statistical analysis (20 trials) shows that the best system raises the spot by $225 \%$, the worst by $1900 \%$ with an average increase of $850 \%$. The RMS spot size after manufacturing will be less than 63 um with a probability of $90 \%$, less than 34 um with $50 \%$, and $10 \%$ of the lens will have a RMS spot size less than 15 um.

This tolerance analysis shows that the Human based design is more robust than the EA lens. The EA lens is mostly sensitive to manufacturing errors (thickness, wedge). If both lenses are built, there is a $10 \%$ probability of obtaining the exact same result as in the laboratory. But the human based design will be produced with a probability of $90 \%$ with a spot size twice as small as the EA design. It should be recalled here that the nominal EA design spot size was smaller by a factor of 2 . So the large margin of the EA design was not enough to guarantee the manufacturing process.

These results are not very surprising from an optical design point of view. The manufacturing and the assembly tolerances are very sensitive to lens bending and overall lens shape. During the optimization process, the optical designer can discard bad designs based on his experience. But, it should be stressed that no constraints or measures were used during the EA process to guide the optimization process towards the generation of robust solutions. The shape of the lens is somewhat difficult to put into numbers, it is much more related to the personal feeling of the optical designer. Lens bending, however, could easily be taken into account by the EA optimization process, for example by formulating some kind of manufacturing and assembly tolerances as an optimization criterion, in order to generate solutions that are both robust and of good quality. Indeed, as we previously indicated with the simultaneous optimization of lens quality and $\operatorname{cost}^{8}$, EA can deal well with this kind of multi-objective optimization problem.

\section{CONCLUSION}

In the first part of the paper we have discussed the monochromatic quartet design. The four lens systems presented were essentially very good solutions. Both lenses from experts using optical design software, and from EA optimization processes have provided notable results. We can observe that the EA process found solutions better than the best solution found by experts.

The second part about the imaging lens problem is very interesting. The nominal results showed that the EA lens was better than the expert design by a factor of almost two. Even though both lenses respect the problem specifications with different safety margins, a manufacturing and assembly tolerance analysis has shown that the 
EA lens was up to four times more sensitive than the lens produced by the human expert, thus losing its nominal advantage. This is not very surprising; tolerances are very sensitive to lens bending and overall lens shape, which was not taken into account by the EA.

In conclusion, lens system design is a very complex process in which it would be surprising that human expertise could be completely removed. Nevertheless, by being human competitive in terms of nominal results, EA have shown their great potential. The next step is now to find a way to include other objectives such as ray bending, or even a sensitivity analysis, into the EA process, in order to design optics better suited for manufacturing.

\section{ACKNOWLEDGMENTS}

Simon Thibault would like to thanks INO researchers, Min Wang and Jonny Gauvin for fruitful discussion. This research was supported by a NSERC-Canada scholarship to C. Gagné and a NSERC-Canada grant to M. Parizeau.

\section{REFERENCES}

1. Berlyn Brixner, Lens design and local minima, Appl. Opt. 20,384-387 (1981).

2. Zemax, Optical Design Program, User's Guide chapter 14.

3. Doron Sturlesi and Donald C. O'Shea. Future of global optimization in optical design. In Proceedings of International Lens Design Conference 1990 ILDC'90, volume 1354, pages 54-68. Proc. of SPIE, 1990.

4. Gregory W. Forbes and Andrew E. W. Jones. Towards global optimization with adaptive simulated annealing. In Proceedings of International Lens Design Conference 1990 ILDC'90, volume 1354, pages 144-153. Proc. of SPIE, 1990.

5. Gregory K. Hearn. Practical use of generalized simulated annealing optimization on microcomputers. In Proceedings of International Lens Design Conference 1990 ILDC'90, volume 1354, pages 186-193. Proc. of SPIE, 1990.

6. Masaki Isshiki. Global optimization with escape function. In Proceedings of International Optical Design Conference 1998, volume 3482 of Proc. of SPIE, pages 104-109,1998.

7. Julie Beaulieu, Christian Gagné, and Marc Parizeau. Lens system design and reengineering with evolutionary algorithms. In Genetic and Evolutionary Computations COnference (GECCO) 2002, pages 155-162, New York, NY, USA, 2002.

8. Christian Gagné, Julie Beaulieu, Marc Parizeau and Simon Thibaullt, Human-competitive Lens System Design with Evolutionary Algorithms, submitted to IEEE Transactions on Evolutionary Computations, July 2005.

9. Donald C. O'Shea. The monochromatic quartet: A search for the global optimum. In Proceedings of International Lens Design Conference 1990, volume 1354 of Proc. of SPIE, pages 548-554, 1990.

10. Hans-Georg Beyer, Hans-Paul Schwefel, Evolution Strategies - A Comprehensive Introduction, Natural Computing, vol. 1, num. 1, pages 3 -52, may 2002.

11. Nikolaus Hansen. The CMA evolution strategy: A tutorial. http://www.bionik.tuberlin.de/user/niko/cmatutorial.pdf, 2004.

12. www.zemax.com 